\title{
"Tradamixina TP" Compound in Monotherapy for Three Months in Patients with LUTS: Observational Study on Improvement of Urinary Symptoms and Sexual Function in Men
}

\author{
Trama Francesco $^{1}\left({ }^{\circ}\right.$, Riccardo Filippo $^{1}$, Romeo Giuseppe ${ }^{2}$, Ruffo Antonio $^{1}$ \\ ${ }^{1}$ Department of Urology-University Federico II of Naples, Via S. Pansini, Naples, Italy \\ ${ }^{2}$ AORN Cardarelli Napoli, Italy \\ Email: francesco.trama@unina.it
}

How to cite this paper: Francesco, T., Filippo, R., Giuseppe, R. and Antonio, R. (2019) "Tradamixina TP" Compound in Monotherapy for Three Months in Patients with LUTS: Observational Study on Improvement of Urinary Symptoms and Sexual Function in Men. Health, 11, 621-629. https://doi.org/10.4236/health.2019.116052

Received: May 5, 2019

Accepted: May 31, 2019

Published: June 3, 2019

Copyright $\odot 2019$ by author(s) and Scientific Research Publishing Inc. This work is licensed under the Creative Commons Attribution International License (CC BY 4.0).

http://creativecommons.org/licenses/by/4.0/

\begin{abstract}
Introduction: Benign Prostatic Hypertrophy (BPH) and Lower Urinary Tract Symptoms (LUTS) frequently affect the male population after the age of 50 . Moreover, it is well known that there is a correlation of these symptoms with Erectile Dysfunction (ED) that can reach a frequency of roughly $52 \%$ in men aged between 40 and 70 years old. In this study, we aimed at evaluating the effect of a new natural compound "Tradamixina TP" (composed of $80 \mathrm{mg}$ of the algae Ecklonia Bicyclis, $100 \mathrm{mg}$ of Tribulus terrestris and $100 \mathrm{mg}$ of Chitosan Water-soluble oligosaccharide $320 \mathrm{mg}$ of Serenoa repens) in the improvement of Lower Urinary Tract Symptoms (LUTS). Materials \& Methods: One-hundred patients with an average age of 64 years old and who were not taking any medication or phytotherapy for prostatic hypertrophy or erectile dysfunction were recruited in the study. All participants were subjected to serum prostate antigen (PSA) and uroflowmetry measuring maximal urine flow/sec (Qmax), at baseline and after 12 weeks. In addition, patients were asked to take the Tradamixina TP for 3 months and to complete at the beginning and end of the treatment the following questionnaires: 1) the IPSS investigating the level of LUTS, and 2) the IIEF-15 investigating the erectile dysfunction. Results: After the treatment, the IPSS scores were lower than those before the treatment. Moreover, a significant effect was found for the IIEF erectile subdomain with scores that were significantly higher at 12 weeks than at baseline. A similar result was found for the IIEF overall subdomain. A decrease of total serum PSA was also observed. Conclusions: After 3 months of daily treatment, the new compound "Tradamixina TP" improved patients' sexual function, LUTS and led also to a decrease in serum PSA levels.
\end{abstract}




\section{Keywords}

Lower Urinary Tract Symptoms, Benign Prostatic Hyperplasia, Tradamixina

TP, Erectile Dysfunction, Tribulus Terrestris, Algae Ecklonia Bicyclis,

Chitosan Water-Soluble Oligosaccharide

\section{Introduction}

Lower Urinary Tract Symptoms (LUTS) are extremely common in men and increase with age [1]. Numerous studies have shown that prostate enlargement, in some subjects, starts early from the third decade of life and increases with the age [2].

LUTS can be broadly divided into storage symptoms (urgency, frequency, urgency incontinence), voiding symptoms (hesitancy, slow stream) and post-voiding symptoms (dripping, feeling of incomplete emptying) [3].

As shown by Abrams and co-workers [4], the most common urinary symptom reported by a significant number of men was altered filling (51.3\%) followed by altered emptying (25.7\%) or the altered end of urination (16.9\%) [4].

Benign Prostatic Hypertrophy (BPH) is the pathological process that contributes the most to the development of LUTS in adult men, although, it is now clear that a significant portion of LUTS is due to a detrusorial dysfunction. Due to cervical urethral alteration, obstructed urinary flow generates neuronal alterations that contribute at different degrees to the development of the symptoms [5].

Histopathologically, BPH is characterized by a numerical increase in epithelial and stromal cells with localized nodular growth preferably at the level of the defined transitional prostatic periurethral area. An involvement of hyperplasia of the basal cells of the prostate ducts was found at the cellular level with an increase in the stromal component and smooth muscle cells, increased deposition of extracellular matrix and deposition of starchy bodies and calcifications [6]. Periurethral nodules in $\mathrm{BPH}$ compress the urethra and this may cause urodynamic obstruction. The obstruction that, in turn, causes LUTS can generate secondary changes that can lead to detrusor muscle hypertrophy, development of recurrent urinary infections, conspicuous post-urinary tract residue and deterioration of the upper urinary tract.

Sympathetic signaling pathways are important in the pathophysiology of LUTS. The sympathetic system is responsible for the expulsion of prostate fluid into the urethra during ejaculation, and the parasympathetic system (cholinergic nerves) increases the rate of secretion [7]. In addition, the neuronal system has been shown to regulate prostate function and growth [8]. Neuronal systems with effects on the prostate include the adrenergic alpha [9], adrenergic beta [10], cholinergic [11], enkephalinergic [12], peptidergic and nitrergic system [13].

As concerns receptors, the presence of alpha 1 receptor is the first factor behind urethral resistance, causing an obstacle to the correct outflow of urine. 
Pharmacological treatment with drugs called alpha-blockers is the first-line therapeutic strategy for $\mathrm{BPH}$.

Furthermore, tropism and the secretory activity of the epithelial cells of the prostate are under the control of androgenic hormones, and, in particular, of a steroid called dihydrotestosterone (DHT), which is produced by the enzymatic reduction of testosterone by 5 alpha-reductase.

The longest-established phytotherapy used to contrast BPH involves the use of Serenoa repens, which it has already been approved in France and Germany for medical treatment [14]. Serenoa repens has shown to be efficient in inhibiting the activity of the enzyme 5 alpha-reductase as well as the bond with the DHT receptor. In addition, Serenoa repens has anti-inflammatory effects and increases the availability of sex hormone-binding globulins [14] [15].

Several authors have focused their attention on the role of chronic inflammation at the prostate level and on how it generates immunological and hormonal events within the gland that, if chronical can lead to the alteration of the cell population at the prostate gland level with subsequent tissue remodeling [16]. In this way, nodules of adenoma are generated. These nodules provoke inflammation, the increase in size of the gland and prostatic hypertrophy. It has been shown that prostate inflammation occurs early in the life span of the disease. Therefore, an early medical intervention can help to delay the onset of the symptoms. In this view, a treatment based on the use of Serenoa repens can have a positive effect on LUTS.

Moreover, BPH is increasingly associated with new onset Erectile Dysfunction (ED). Gacci and co-workers [17] have identified four pathogenetic theories behind ED/LUTS: A reduced NOS-NO signal; the theory of autonomic hyperactivity; the activation of Rho-kinase/endothelin and Pelvic atherosclerosis. These theories, together or separately, can lead to dysfunctions at the level of the prostate, corpora cavernosa, urethra and bladder due to alterations of the nerve tissue and endothelium, the altered mechanism of muscle relaxation/contractility, and the reduced blood flow with consequent tissue damage, caused by hypoxia [17]. Accordingly, a new natural compound called "Tradamixina TP" (composed by algae Ecklonia bicyclis, Tribulus terrestris and Chitosan Water-soluble oligosaccharide, Serenoa repens) showed a general improvement of the sexual activity [18].

In the present study, we aimed at evaluating the effect of the new natural compound "Tradamixina TP" made of the algae Ecklonia bicyclis, Tribulus terrestris Chitosan Water-soluble oligosaccharide and Serenoa repensorder to improve LUTS. To do this, patients were asked to take the Compound for 3 months and to complete, at the baseline and after 12 weeks, two questionnaires: The International Prostate Symptom Score (IPSS) investigating the level of LUTS; and b) the International Index Erectile Function (IIEF-15) investigating the erectile dysfunction through IIEF erectile, IIEF sexual, IIEF orgasmic, IIEF intercourse, and IIEF overall subdomains. 


\section{Methods and Materials}

One-hundred adult patients (Age range 45 - 80; Median age 64; $\mathrm{SD}=11.02$ ) took part in the study, following written consent for their participation. All patients were recruited from the Department of Urology, Federico II University of Naples (Italy). Participants' recruitment and testing were in conformity with the Helsinki Declaration of 1974. The experimenters who run the experiment and all the participants were blind to the hypotheses of the study. All participants, who were diagnosed with LUTS/BPH for $>6$ months at screening, with IPSS (International Prostatic Symptoms Score) $\geq 13$ and maximum urinary flow rate (Qmax) $\geq 4$ to $\leq 15 \mathrm{ml} / \mathrm{s}$, were included in the dataset.

Exclusion criteria were: The assumption of any medication or phytotherapy for LUTS or ED (alpha receptor antagonists, Serenoa repens, dutasteride, finasteride, PDE 5 inhibitors), the presence of hyper- and hypothyroidism, of neurogenic syndromes (Multiple sclerosis, multiple atrophy, Parkinson's disease, tumors, stroke, disk disease, spinal cord disorders, polyneuropathy, uraemia), of lower pelvic surgery (oncological pelvic surgery, lower urinary and genital tract surgery), or prostate cancer.

High IPSS illustrate IPSS and IIEF-15 questionnaires, respectively. High IPSS scores correspond to the severity of the symptoms. By contrast, low IIEF-15 scores correspond to the severity of the symptoms.

\subsection{Procedure}

All participants were subjected to serum prostate antigen (PSA) and uroflowmetry measuring maximal urine flow/sec (Qmax), at baseline and after 12 weeks. In addition, patients were asked to take the "Tradamixina TP" and to complete the IIEF-15 and the IPSS questionnaires, at the beginning and end of the treatment. The questionnaires were administered in a counterbalanced order across participants. The testing phase took about 30 minutes.

\subsection{Data Analysis}

Separate ANOVAs were performed on the means of the scores at the baseline and after the treatment for the IIEF-15 (and its five subscales) and the IPSS questionnaires. The magnitude of the significant effects was indicated by a partial eta squared $\left(\eta p^{2}\right)$.

\section{Results}

The ANOVA revealed a significant difference $\left(\mathrm{F}(1109)=17.164, \mathrm{p}<0.05, \eta \mathrm{p}^{2}=\right.$ $0.04)$ of the values of PSA being lower after 12 weeks $(\mathrm{M}=2.77, \mathrm{SD}=2.07)$ than at baseline $(\mathrm{M}=3.33, \mathrm{SD}=1.48)$. By contrast, no significant difference emerged concerning $\mathrm{Qmax}\left(\mathrm{F}(1.99)=1.581, \mathrm{p}=0.21, \eta \mathrm{p}^{2}=0.01\right)$.

Concerning the IPSS questionnaire, after 12 weeks the IPSS scores were significantly lower $(M=18.22, S D=10)$ than those before the treatment $(M=$ $22.52, \mathrm{SD}=7.58): \mathrm{F}(1.99)=12.463, \mathrm{p} \leq 0.001, \eta \mathrm{p}^{2}=0.11$ (Figure 1 ). 


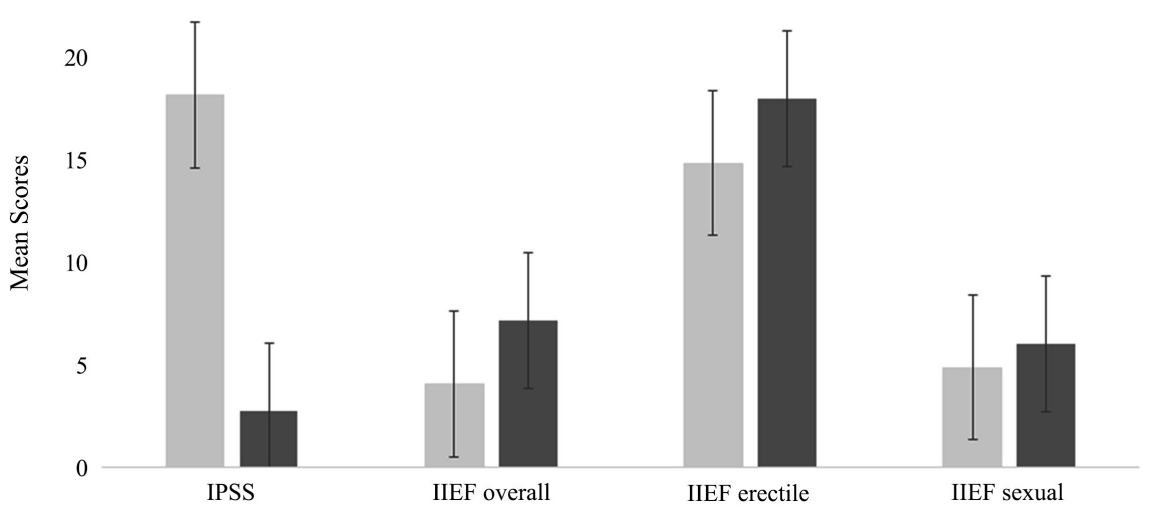

Figure 1. The Figure illustrates the changes of the scores at the baseline and after 12 weeks of treatment for the IIEF-15 (overall, erectile and sexual subscales) and the IPSS questionnaires. Error bars represent standard errors.

A significant effect also emerged for some subdomains of the IIEF-15 questionnaire. Specifically, after 12 weeks the IIEF overall scores $(\mathrm{F}(1109)=3.917, \mathrm{p}$ $\left.\leq 0.05, \eta p^{2}=0.08\right)$ were significantly higher $(\mathrm{M}=7.17, \mathrm{SD}=2.45)$ than the scores at baseline $(\mathrm{M}=4.11, \mathrm{SD}=2.80)$. As shown by Figure 1 , a similar trend was also found for the subdomains IIEF erectile $\left(\mathrm{F}(1109)=6.467, \mathrm{p} \leq 0.05, \eta p^{2}\right.$ $=0.06$; IIEF erectile baseline score: $\mathrm{M}=14.90, \mathrm{SD}=8.80$; IIEF erectile 12-week score: $\mathrm{M}=18.07, \mathrm{SD}=8.55)$ and IIEF sexual $\left(\mathrm{F}(1109)=3.467, \mathrm{p} \leq 0.05, \eta p^{2}=\right.$ 0.07; IIEF sexual baseline score: $\mathrm{M}=4.90, \mathrm{SD}=1.70$; IIEF sexual 12-week score: $\mathrm{M}=6.09, \mathrm{SD}=1.51)$. Finally, no significant difference emerged for the remaining subdomains, IIEF orgasmic $(\mathrm{F}<1)$ and IIEF intercourse $(\mathrm{F}<1)$.

\section{Discussions}

The natural compound used in this study, characterized by Tradamixina TP (composed of the algae Eckloni abicyclis, the plant Tribulus terrestris and the water-soluble oligosaccharide chitosan and Serenoa repens) was shown to be associated with the improvement in LUTS and of penile erection and libido.

This finding can be ascribed to the characteristics of the individual components of the "Tradamixina TP" compound. Indeed, the alga Ecklonia bicyclis, thanks to its anti-inflammatory and anti-oxidant effects, suppresses the production of prostaglandin E2 and oxygenase cycle $2(\mathrm{COX}-2)$ in a dose-dependent manner, without causing any cytotoxicity. Moreover, previous studies have shown that the algae Eckloni abicyclis reduces the formation of pro-inflammatory cytokines, such as interleukin (IL-1 beta) and the tumor necrosis factor TNFalfa, by stimulating the production of reactive oxygen species (ROS) [19].

The compound Chitosan Water-soluble oligosaccharide which acts both on the non-adrenergic and non-cholinergic cell system (NANC) and on the endothelial cell system was a strong stimulation of nNOS. Besides, Tribulus terrestris has an effect on erectile function due to its ability to activate the neuronal NO 
synthase pathways that act at the endothelial level of the corpora cavernosa [20]. Some authors have reported that Tribulus terrestris also increases the serum levels of total testosterone [21]. However, evidence is not converging. For example, Santos and colleagues [22] argue that there is no difference between Tribulus and placebo, either in the improvement of ED or in the increase in serum testosterone levels. By contrast, Roaiah and co-workers [23] assert that Tribulus may be considered as a therapeutic option for ED patients with PADAM syndrome (partial androgen deficiency in aging male) because it can increase both the IIEF-15 score and serum testosterone levels. A recent review has also reported that Tribulus has a positive effect on both libido and erectile function, although data on its androgenic properties are still partial and not conclusive [24].

Research has also observed that both Chitosan Water-soluble oligosaccharide and Tribulus terrestris may have effects on the inhibition of pro-inflammatory mediators.

$\mathrm{BPH}$ is known to be associated with changes in innervation as well as in the production and release of pro-inflammatory mediators in the central area of the prostate [25] and in the expression of Neuronal Nitroxide Synthase (nNOS), which regulates blood pressure, smooth muscle relaxation and vasodilatation via peripheral nitrate nerves. These nerves are of particular importance in the relaxation of the corpus cavernosum and during the erection of the penis.

Compared to the normal tissue, in patients affected by $\mathrm{BPH}$, it has been observed that the enzyme nNOS tends to decrease in the transition zone of the central tissue where the adenoma is present. On the other hand, it has also been reported that Inducible Nitroxide Synthase (iNOS), produced as a result of the stimulation of cytokines, inflammatory and free radicals, selectively increases in the stroma of patients affected by BPH [26]. This observation is probably due to the increased pro-inflammatory conditions in BPH [27]. In fact, inducible nitroxide synthase (iNOS) is expressed in a variety of cell types, particularly in inflammation promoting cells, in response to different pro-inflammatory stimuli.

Consistently, a recent study [28] has reported that the inhibition of NOS-I can be achieved by activating phospholipase A2 (cPLA2), an enzyme belonging to the PLA2 enzyme family.

On the other hand, neuronal nitroxide synthase (nNOS) requires critical levels of Arachidonic Acid (AA), generated by the phospholipase constitutive A2 (PLA2), which, in turn, promotes the activation of the tyrosine kinase-dependent phosphorylation and inhibition of the NOS constitutive.

Therefore, the AA produced at the earliest stages of the inflammatory response is a likely critical signal that switches the regulation of NO formation from a physiological (i.e. nNOS-mediated) to a pathological (i.e. iNOS-mediated) condition. The second phase of the inflammatory response is often accompanied by the onset of deleterious effects where a critical role is played by iNOS-derived NO (directly or indirectly, i.e. through peroxynitrite formation) and AA cascade 
products [29].

As regards the Serenoa repens, it improves LUTS when used over the long-term thanks to its phytotherapeutic properties, i.e. its anti-androgen, anti-inflammatory, antiproliferative and pro-apoptotic features. However, a recent Cochrane meta-analysis [30] reports of no significant advantage in taking Serenoa for the IPSS questionnaire and Qmax.

Finally, encouraging data have been recently reported on the use of Serenoa repens in combination with alpha-blocking drugs compared to the use of alpha-blockers alone in the improvement of the urinary symptoms [31].

\section{Conclusion}

The daily treatment with the new compound "Tradamixina TP" (composed of $100 \mathrm{mg}$ Chitosan Water-soluble oligosaccharide $80 \mathrm{mg}$ of algae Ecklonia Bicyclis, $100 \mathrm{mg}$ of Tribulus terrestris and $100 \mathrm{mg}$ of plus $320 \mathrm{mg}$ of Serenoa repens), which was administered for 3 months, improved patients' sexual function, the urinary symptoms and also led to a decrease in serum PSA levels.

\section{Conflicts of Interest}

The authors declare no conflicts of interest regarding the publication of this paper.

\section{References}

[1] Rosen, R., Altwein, J., Boyle, P., et al. (2003) Lower Urinary Tract Symptoms and Male Sexual Dysfunction: The Multinational Survey of the Aging Male (MSAM-7). European Urology, 44, 637-649. https://doi.org/10.1016/j.eururo.2003.08.015

[2] Berry, S.J., Coeffy, D.S., Walsh, P.C., et al. (1984) The Development of Human Benign Prostatic Hyperplasia with Age. Journal of Urology, 132, 474-479. https://doi.org/10.1016/S0022-5347(17)49698-4

[3] Irwin, D.E., Milsom, I., Hunskaar, S., et al. (2006) Population-Based Survey of Urinary Incontinence, Overactive Bladder, and Other Lower Urinary Tract Symptoms in Five Countries: Results of the EPIC Study. European Urology, 50, 1306-1315. https://doi.org/10.1016/j.eururo.2006.09.019

[4] Abrams, P., Cardozo, L., Fall, M., et al. (2003) The Standardisation of Terminology in Lower Urinary Tract Function: Report from the Standardisation Sub-Committee of the International Continence Society. Urology, 61, 37-49. https://doi.org/10.1016/S0090-4295(02)02243-4

[5] Bruschini, H., Schmidt, R.A. and Tanagho, E.A. (1978) Neurologic Control of Prostatic Secretion in the Dog. Investigative Urology, 15, 288-290.

[6] Isaacs, J.T. (1994) Etiology of Benign Prostatic Hyperplasia. European Urology, 25, 6-9. https://doi.org/10.1159/000475324

[7] Smith, E.R. and Lebeaux, M.I. (1970) The Mediation of the Canine Prostatic Secretion Provoked by Hypogastric Nerve Stimulation. Investigative Urology, 7, 313-318.

[8] Burnett, A.L., Maguire, M.P., Chamness, S.L., Ricker, D.D., Takeda, M., Lepor, H. and Chang, T.S. (1995) Characterization and Localization of Nitric Oxide Synthase in the Human Prostate. Urology, 45, 435-439.

https://doi.org/10.1016/S0090-4295(99)80012-0 
[9] Higgins, J.R. and Gosling, J.A. (1989) Studies on the Structure and Intrinsic Innervation of the Normal Human Prostate. The Prostate. Supplement, 2, 5-16. https://doi.org/10.1002/pros.2990150503

[10] Tsujii, T., Azuma, H., Yamaguchi, T. and Oshima, H. (1992) A Possible Role of Decreased Relaxation Mediated by Beta-Adrenoceptors in Bladder Outlet Obstruction by Benign Prostatic Hyperplasia. British Journal of Pharmacology, 107, 803-807. https://doi.org/10.1111/j.1476-5381.1992.tb14527.x

[11] Caine, M., Raz, S. and Zeigler, M. (1975) Adrenergic and Cholinergic Receptors in the Human Prostate, Prostatic Capsule and Bladder Neck. British Journal of Urology, 47, 193-202. https://doi.org/10.1111/j.1464-410X.1975.tb03947.x

[12] Jungblut, T., Aumuller, G., Malek, B. and Melchior, H. (1989) Age Dependency and Regional Distribution of Enkephalinergic Nerves in Human Prostate. Urologia Internationalis, 44, 352-356. https://doi.org/10.1159/000281539

[13] Lange, W. and Unger, J. (1990) Peptidergic Innervation within the Prostate Gland and Seminal Vesicle. Urological Research, 18, 337-340. https://doi.org/10.1007/BF00300783

[14] Lepor, H. and Shapiro, E. (1984) Characterization of Alpha1 Adrenergic Receptors in Human Benign Prostatic Hyperplasia. Journal of Urology, 132, 1226-1229. https://doi.org/10.1016/S0022-5347(17)50110-X

[15] Di Silverio, F., D’Eramo, G., Lubrano, C., Flammia, G.P., Sciarra, A., Palma, E., et al. (1992) Evidence That Serenoa repens Extract Displays an Antiestrogenic Activity in Prostatic Tissue of Benign Prostatichypertrophy Patients. European Urology, 2, 309-314. https://doi.org/10.1159/000474863

[16] Briganti, A., Capitanio, U., Suardi, N., et al. (2009) Benign Prostatic Hyperplasia and Its Aetiologies. European Urology Supplements, 8, 865-871. https://doi.org/10.1016/j.eursup.2009.11.002

[17] Gacci, M., Eardley, I. and Giuliano, F. (2011) Critical Analysis of the Relationship between Sexual Dysfunctions and Lower Urinary Tract Symptoms Due to Benign Prostatic Hyperplasia. European Urology, 60, 809-825. https://doi.org/10.1016/j.eururo.2011.06.037

[18] Iacono, F., Stanojevic, N., Skrodzka, M., et al. (2015) Improving Male Sexual Function with a Nutraceutical Composite Containing Tribulus terrestris, Alga Eisenia bicyclis and Chitosan Oligosaccharide Water Soluble: A Multicentric, Randomized, Placebo-Controlled Study. The Journal of Sexual Medicine, 12, 294-381. https://doi.org/10.1111/jsm.12956

[19] Jung, W.-K., Heo, S.-J., Jeon, Y.-J., et al. (2009) Inhibitory Effects and Molecular Mechanism of Dieckol Isolated from Marine Brown Alga on Cox-2 and iNOS in Microglial Cells. Journal of Agricultural and Food Chemistry, 57, 4439-4446. https://doi.org/10.1021/jf9003913

[20] Do, J., Choi, S., Choi, J. and Hyun, J.S. (2013) Effects and Mechanism of Action of a Tribuusterrestris Extract on Penile Erection. Korean Journal of Urology, 54, 183-188. https://doi.org/10.4111/kju.2013.54.3.183

[21] Russo, A., Maisto, E., Romis, L., et al. (2016) Use of a Natural Compound Made of Eisenia bicyclis Seaweed, Tribulus terrestris and Water-Soluble Chitosan Oligosaccharide, in Male Sexual Asthenia with Mild or Mild-Moderate Erectile Dysfunction and Serum Testosterone Levels at the Lower Limit of Normal. Health, 8, 1668-1678. https://doi.org/10.4236/health.2016.815162

[22] Santos, C.A., Reis, L.O., Destro-Saade, R., Luiza-Reis, A. and Fregonesi, A. (2014) Tribulus terrestris versus Placebo in the Treatment of Erectile Dysfunction: A 
Prospective, Randomized, Double Blind Study. Actas Urologicas Espanolas, 38, 244-248. https://doi.org/10.1016/j.acuroe.2014.03.009

[23] Roaiah, M.F., El Khayat, Y.I., Gamal El Din, S.F. and Abd El Salam, M.A. (2016) Pilot Study on the Effect of Botanical Medicine (Tribulus terrestris) on Serum Testosterone Level and Erectile Function in Aging Males with Partial Androgen Deficiency (PADAM). Journal of Sex and Marital Therapy, 7, 297-301. https://doi.org/10.1080/0092623X.2015.1033579

[24] Neychev, V. and Mitev, V. (2016) Pro-Sexual and Androgen Enhancing Effects of Tribulus terrestris L.: Fact or Fiction. Journal of Ethnopharmacology, 179, 345-355. https://doi.org/10.1016/j.jep.2015.12.055

[25] Chapple, C.R., Crowe, R., Gilpin, S.A., Gosling, J. and Burnstock, G. (1991) The Innervation of the Human Prostate Gland-The Changes Associated with Benign Enlargement. Journal of Urology, 146, 1637-1644. https://doi.org/10.1016/S0022-5347(17)38203-4

[26] Nickel, J.C., Downey, J., Young, I. and Boang, S. (1999) Asymptomatic Inflammation and/or Infection in Benign Prostatic Hyperplasia. BJU International, 84, 976-981.

[27] Elkahwaji, J.E. and Zhong, W. (2007) Hopkins Chronic Bacterial Infection and Inflammation Incite Reactive Hyperplasia in a Mouse Model of Chronic Prostatitis. Prostate, 67, 14. https://doi.org/10.1002/pros.20445

[28] Leslie, C.C. (1997) Properties and Regulation of Cytosolic Phospholipase A2. The Journal of Biological Chemistry, 272, 16709-16712. https://doi.org/10.1074/jbc.272.27.16709

[29] Mariotto, S., Suzuki, Y., Persichini, T., Colasanti, M., Suzuki, H. and Cantoni, O. (2007) Cross-Talk between No and Arachidonic Acid in Inflammation. Current Medicinal Chemistry, 14, 1940-1944. https://doi.org/10.2174/092986707781368531

[30] Tacklind, J., et al. (2012) Serenoa repens for Benign Prostatic Hyperplasia. Cochrane Database of Systematic Reviews, 12, Cd001423.

https://doi.org/10.1002/14651858.CD001423.pub3

[31] Ryu, Y.W., et al. (2015) Comparison of Tamsulosin plus Serenoa repens with Tamsulosin in the Treatment of Benign Prostatic Hyperplasia in Korean Men: 1-Year Randomized Open Label Study. Urologia Internationalis, 94, 187. https://doi.org/10.1159/000366521 